\title{
A key role of CUG-binding protein 1 in HSC activation and liver fibrogenesis revealed by fraxinellone
}

Xingxin $\mathrm{Wu}^{1,2}$, Xudong $\mathrm{Wu}^{1,2}$, Yang Sun ${ }^{1,2}$, Xuefeng $\mathrm{Wu}^{1,2}$, Qiang $\mathrm{Xu} \mathrm{u}^{1,2}$

${ }^{\text {IS }}$ chool of Life Sciences, Nanjing University, China, ${ }^{2}$ State Key Laboratory of Pharmaceutical Biotechnology, China

Excessive activation of hepatic stellate cells (HSCs) is a key step in liver fibrogenesis. Here we report that CUG-binding protein 1 (CUGBP1) expression is elevated in HSCs and positively correlates with liver fibrosis severity in human liver biopsies. Transforming growth factor beta (TGF- $\beta$ ) selectively increases CUGBP1 expression in cultured HSCs in a p38 mitogen-activated protein kinase (MAPK)-dependent manner. Knockdown of CUGBP1 inhibits alpha smooth muscle actin ( $\alpha$-SMA) expression and promotes interferon gamma (IFN- $\gamma$ ) production in HSCs in vitro. We further show that CUGBP1 specifically binds to the 3' untranslated region (UTR) of human IFN- $\gamma$ mRNA and promotes its decay. In mice, knockdown of CUGBP1 alleviates, whereas its overexpression exacerbates, bile duct ligation (BDL)-induced hepatic fibrosis. Therefore, CUGBP1-mediated IFN- $\gamma$ mRNA decay is a key event for profibrotic TGF- $\beta$-dependent activation of HSCs, and inhibiting CUGBP1 to promote IFN $-\gamma$ signaling in activated HSCs could be a novel strategy to treat liver fibrosis. 